\title{
Improving the Effectiveness of Protective Means through Modelling the Initial Kinematic Parameters of the Fragmentation Field
}

\author{
Stancho P. Petkov, Petko S. Petkov, and Adelina D. Tumbarska
}

\begin{abstract}
The purpose of this article is to develop a mathematical model describing the process of forming the initial kinematic parameters of the fragmentation field. Efforts to increase the effectiveness of ammunitions have been explored, namely increasing the area of damage, by using system for continuous initiation of the explosive substance. The results obtained can be used in the development of ammunitions designed for countering terrorist and other threats as components of critical infrastructure protection systems.
\end{abstract}

Index Terms-Fragmentation Warhead; Initial Kinematic Parameters of Fragmentation Field; Impact-Wave Picture.

\section{INTRODUCTION}

The objects of critical infrastructure in today's dynamic, interconnected and interdependent world are increasingly vital factor in the development of industrialized countries and their economies. Over the past decade, the efficiency of critical infrastructure protection has become a priority issue for the countries to respond to increasing risks of terrorism.

Modern systems for infrastructure perimeter protection usually have two main zones with different levels of risk warning zone and protected zone; the warning zone is divided into two sub-areas - for surveillance and warning. The outermost zone is intended to alert the staff when people or vehicle approaches, the average - for assessing the intruder's intent and the threat, and warning the offender by various means. The inner zone is focused on counteracting the threat [1]. Variety of systems for protection of key infrastructure objects, using sophisticated methods and means of monitoring, detecting and identifying intruders, are developed at the IMSETHAC-BAS, including non-lethal and lethal means to counteract hostile acts [2]. Means with focused and unfocused impact are created for using in cases of particularly serious threats when physical destruction of the offender (people or technical equipment) can not be avoided. Each protection system configuration depends on the specificity and importance of the protected object and is consistent with the degree of risk and types of potential threats, providing the functioning of the system as a whole, as well as of its subsystems and components, in accordance

Published on April 4, 2018.

The authors are with Department "Technologies and systems for protection" at the Institute of Metal Science, Equipment and Technology with Hydro- and Aerodynamics Centre "Acad. Angel Balevski" - Bulgarian Academy of Sciences (IMSETHAC-BAS), Sofia, Bulgaria. S. P. Petkov is Professor, Dr. Eng. (e-mail: s_petkov63@abv.bg); P. S. Petkov is PhD student, MSc. Ec. (e-mail: petko_st_petkov@abv.bg); A. D. Tumbarska is Assistant-Professor, Dr. Eng. (e-mail: y.toumbarski@ims.bas.bg). with the specially developed quality criteria [3], [4].

The theory of the destruction and ejection of plates under the impact of explosive detonation products (DP) at the present time is a separate section in the physics of the explosion, which is often used as explosive ballistics.

As a result of the development of experimental techniques and the widespread application of non-standard methods of gas dynamics, dynamic plasticity, solid state physics and fracture theory, theoretical and experimental material on this issue has been accumulated and significant number of research and scientific papers have been published.

One of approaches to improvement of the fragmentation warhead of different purpose ammunitions is increasing the efficiency at the expense of a predetermined distribution of the fragments field within the area of action.

\section{DeVelopment Of Mathematical Model For DETERMINING THE INITIAL KinEMATIC PARAMETERS OF THE FRAGMENTATION FIELD}

In practice, during the phase of design and impact analysis of the fragmentation ammunitions explosive devices, the first step is solving the problem of determining the initial kinematics parameters of the fragmentation field.

Within this framework, all tasks can be provisionally divided into complex of items connected to: onedimensional movement of the body under the impact of detonation products (DP) [5]; two-dimensional movement under the impact of DP [5].

When developing one-dimensional models, the following are generally used: assumption of instantaneous detonation of the explosive; the energetic, kinematic approach of Gorni-Staniukovic-Pokrovsky; various proposals for the mechanism of distribution of the explosive mass in the direction of scattering - the concept of active masses.

As the analysis of one-dimensional body motion models shows, the one-dimensional radial solutions give the law of movement and the kinetic velocity of infinitely long charges. It is definitely impossible to proceed with charges of a certain length within the one-dimensional task.

On the other hand, the two-dimensional solution of the task allows to adequately determine the process of the body dynamic loading, but the model under consideration is rather complex and its application at the stage of ammunition experimental design, when it is necessary to compare a large number of design variants of technical solutions, is problematic.

In this regard, development of methods for calculating and determining the direction of the fragments velocity 
along the body in a quasi two-dimensional setting, based on the flat sections method, is relevant. It is appropriate to present the process of accelerating the body in two phases:

- wave stage (impact acceleration), depending only on the properties of the explosive, the metal, the body configuration and the means for initiating the explosive;

- ballistic stage, defining the configuration of the bursting charge, the properties of the explosive and the body structural characteristics.

The following assumptions are made during developing the model for the movement of an axis-symmetrical elasticplastic body:

- The fragments scattering is taking place within the framework of the flat sections hypothesis, i.e. the axis coordinate of the section is not changed during the acceleration process;

- The pressure acting on the body is defined as a multiplicative function of pressure at axial and radial scattering of DP;

- The entropy index of the DP is assumed to be equal to $3[6]$;

- The DP pressure is averaged over the cross section and the mass velocity of the DP flow is proportional to the radius;

- The process of scattering the parts of the body is considered in two-stage approximation: the first stage of the body acceleration occurs under the action of the shock wave and the unloading wave (UV), and in the second stage - under the action of expanding DP.

These assumptions are widely used in the course of developing various models at ejecting metal plates by the detonation products and their relevance is proven in the scientific labor of V. A. Odintsov, V. V. Silivanov, T. G. Statsenko and other authors [7], [8].

An axis-symmetrical charge with mass $M=\int_{0}^{h_{3}} m(x) d x$, radius $z_{o}$ and length $h$, on the side surface of which there is a throwing plate, part of the body with thickness $\delta(x)$ is considered. Generally, the initiation of the explosive charge can be continuous or discrete, which also determines the angle of the detonation wave approach to the body, $\propto(x)$.

At the first stage, the parameters of the velocity field of various points under the impact of the falling shock wave and the UV will be determined.

The parameters of the field of velocity in the first stage of the body acceleration under the action of the shock wave and the unloading wave will be determined. Fig. 1 shows an impact-wave pattern in the body in mobile coordinate system associated with the point of intersection of the detonation wave (DW) and the body.

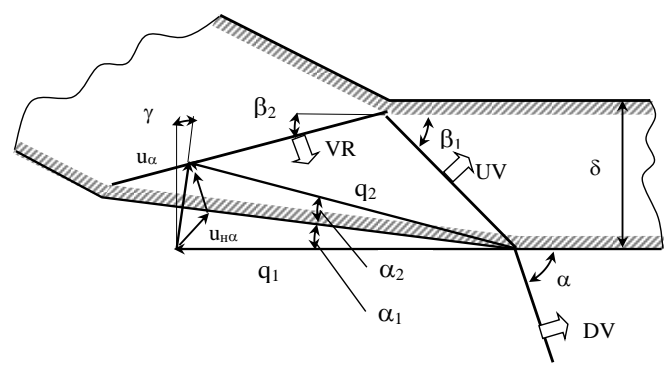

Fig.1. Impact-wave picture in the body at the shock wave approach.
An assumption is made that after completing the first stage the acceleration of the body elements remains unchanged, at the same time the body is unloaded to its initial state, i.e. the condition for velocity continuity of the forming flow and the flow after the transition by the shock wave front and the unloading wave is fulfilled $\left|\overline{q_{1}}\right|=\left|\overline{q_{2}}\right|$. The following ratios are obtained from the shock-wave picture analysis:

$\beta_{1}=\arcsin \frac{C_{0}+\lambda u_{H \propto}}{D} \sin \propto$

$\beta_{2}=\arcsin \left[C_{0}+(\lambda-1) u_{\alpha}\right] \sqrt{1+2 u_{\alpha} \lambda / C_{0}} \frac{\sin \alpha}{D}$

$\gamma=\frac{\alpha_{1}+\alpha_{2}}{2} ; \quad u_{\propto}=2 u_{H \propto} \frac{\cos \left(\beta_{1}-\propto_{1}\right)}{\sin \left(\alpha_{1}\right)} \sin (\gamma)$

where: $C_{0}, \lambda$ are constants of the shock adiabatic of the type $D=C_{0},+\lambda u_{x} ; u_{H \propto}$ - flow velocity in the body behind the shock wave front; $u_{\propto}$ - velocity of the body element after completing the shock-wave stage of acceleration; $\beta_{1} \beta_{2}$ angles of the slope of the shock-wave and sound lines; $\alpha_{1} \alpha_{2}$ - angles of flow divergence behind the shock wave front and the UW.

The following equations are used to determine the shock wave attenuation in the body material [9]:

$p \approx \mathrm{p}_{0}\left(\frac{m_{0}^{\prime}}{M}\right)^{n_{1}} ; u \approx \mathrm{u}_{0}\left(\frac{m_{0}^{\prime}}{M}\right)^{n_{1}}$,

where: $M$ - mass of substances covered by the shock wave; $m_{0}^{\prime}$ - characteristic (active) mass of the explosive.

In the general case, the index $n_{1}$ is determined in the course of the solutions of the differential equations describing the process, but in the first approximation of the adiabatic index $n=7 / 5$ there is an exact analytical solution in which $n_{1}=4 / 3$.

The nature of the shock wave depends on the choice of the characteristic (active) mass of the explosive $m_{0}^{\prime}$, which can be represented with characteristic scale in mass Lagrange coordinates; a mass of the explosive charge which is not covered at the moment of the detonation wave exit at the boundary of ,explosive-body“ contact can be chosen for $m_{0}^{\prime}$. Then:

$M=m_{0}^{\prime} \int_{z_{1}}^{z_{2}} d m=m_{0}+\rho_{n} \delta_{0}$

Taking into account the symmetry of the system and the interaction of the contact boundary between two media with different equations of the state, and assuming an average density of the detonation products $\rho=4 / 3 \rho_{\text {expl }}$, it can be written:

$u_{\propto}=\frac{u_{H \propto}}{\left[1+\frac{\delta}{H^{\prime} \cos \beta_{1}}\left(\frac{3 \rho_{n}}{4 \rho_{\text {expl }}}\right)^{k / 7}\right]^{\frac{2 n}{k+1}}}$,

where $H^{\prime}$ - the characteristic size of the system determining the characteristic (active) mass $m_{0}^{\prime} \quad\left(H^{\prime}=m_{0}^{\prime} / \rho_{\text {expl }}\right)$, where: $\rho_{x}, \rho_{\operatorname{expl}}$ - density of the body material and the explosive substance; $n$ - indicator of symmetry: $n=1,2,3$ 
for flat, cylindrical and spherical shape, respectively.

The initial velocity on the boundary „explosive-body“ at obliquely impact of the DW is determined by solving the task of reflecting the falling DW from the moving wall using the known ratio of the contact surface decomposition and the equation of the body material dynamic contraction.

To determine the pressure at the boundary of the "explosive-body" section, it is necessary to know the dynamic compressibility of the environment under consideration.

If the dynamic compressibility of an arbitrary environment can be expressed by the equation of the type

$p=A \cdot\left(\frac{\rho}{\rho_{0}}\right)^{n}+B$

the pressure $p$ should be determined by equality

$$
\frac{D_{u}}{k+1}\left[\cos \Psi-\sqrt{2 k} \frac{\Pi-1}{\sqrt{(k+1) \Pi+(k-1)}} \cos \varphi\right]=\sqrt{\frac{p_{x}}{\rho_{0}}\left[1-\left(\frac{A}{p_{x}-B}\right)^{\frac{1}{n}}\right]} \cos \beta_{1} .
$$

In this way, calculation of the body element velocity $u_{H}$ allows for calculating the values of the kinematic parameters of the body at the first wave stage, which is also the initial condition for the second gas-dynamic phase [10], [11].

\section{System For Continuous Initiation Of The EXPLOSIVE SUBSTANCE}

Flat deformation of a cylindrical body is considered Fig.2.

The stresses $\sigma_{r}, \sigma_{0}, \sigma_{z}$ are the main ones. Assuming that the material of the shell is inversible, the integral of the continuity equation can be defined as:

$v=v_{a} \frac{a}{r}$

where $v_{a}=\frac{d a}{d t}$ is the speed of movement of the inner surface of the body.

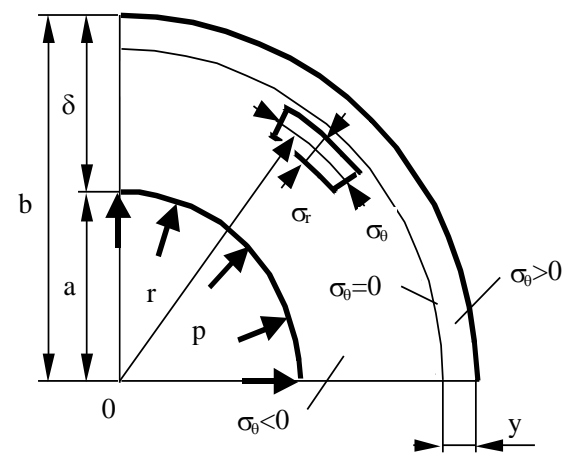

Fig.2. Stressed state of the body under detonation products impact.

By using the equation of the body motion in the shape of Euler and taking into account the continuity of the body material equation and the plasticity conditions of Saint Venant - Tresca, it is obtained: $\frac{d v_{a}}{d t}=\left(\frac{1}{2 a \cdot \ln b / a}-\frac{a}{2 b^{2} \cdot \ln b / a}-\frac{1}{a}\right)\left(\frac{d a}{d t}\right)^{2}+\left[\frac{P}{a \cdot \ln b / a}-\frac{x Y}{a}\right] \cdot \frac{1}{\gamma_{0}}(6)$

where: $\mathrm{b}=\left(b_{0}^{2}-a_{0}^{2}+a^{2}\right)^{1 / 2}$ is current radius of the body on the outer surface; $Y$ - dynamic yield boundary of the body material; $a$ - current radius of the inner surface of the body; $r$ - radial Euler coordinate.

The pressure $P$, is represented in the form of multiplier function

$p=p_{m} \bar{p}(r, t) \bar{p}(x, t)$

where: $\bar{p}(r, t)$ - dimensionless pressure, taking into account the pressure variation in radial direction at the expense of the DP expansion and the movement of the expanding body; $p_{m}$ - amplitude pressure on the detonation wave front.

The pressure can be presented in the form of two components: consisting of the radial expansion of the housing and constituting of the pressure variation accounting for the radial leakage of the DP.

$\bar{p}(r, t)=\left[1-\frac{k-1 u(r, t)}{2 c(x, t)}\right]^{\frac{2 k}{k-1}} \cdot\left(a_{0} / a\right)^{2 k}$,

where $c(x, t)$ - the current local sound speed, $u(r, t)-$ the current value of the speed.

In order to determine the $\overline{p_{x}}(x, t)$, the diagram of the wave process in the DP, shown in Fig.3, is examined. [12].

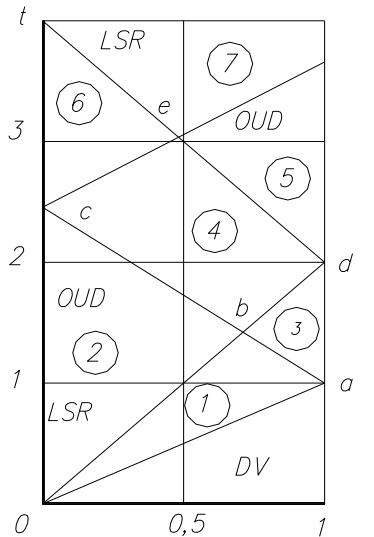

Fig.3. Phase diagram for the movement of the detonation waves and the unloading waves at one-dimensional flow of the DP.

By introducing the relative variables in the time and the path of propagation of the DW and the UW, the expression for the laws of motion of the waves and the relative pressure in the areas under consideration are determined.

The suggested scheme of calculating pressure $\overline{P_{x}}(x, t)$, in the case of a sliding detonation wave typical for longdistance charges, at a distance of approximately 0.5 diameters of charge, the front of the detonation wave can be considered flat.

Thus, solving the system of differential equations (2), together with the dependencies (6) ... (8), and in the body (Table I), allows for determination of the initial kinematical parameters of the fragments bodies. 
TABLE I: INITIAL KINEMATIC PARAMETERS OF THE FRAGMENTS

\begin{tabular}{ccc}
\hline Zone & Law & Pressure \\
\hline \hline 1 & $\bar{x}_{o a}=\bar{t}$ & $\bar{P}=8 / 27(\bar{x} / \bar{t}+0.5)$ \\
\hline 2 & $\bar{x}_{o b}=\bar{t} / 2$ & $\bar{P}=8 / 27$ \\
\hline 3 & $\bar{x}_{a b}=-\bar{t} / 2-\sqrt{\bar{t}} / 2+2$ & $\bar{P}=64 / 27(1 / \bar{t})^{3}$ \\
\hline 4 & $\bar{x}_{b c}=-\bar{t} / 2-\sqrt{\bar{t}} / 2+2$ & $\bar{P}=8 / 27\left[0.5-\frac{\bar{x}-2}{\bar{t}}\right]^{3}$ \\
& $\bar{x}_{b d}=\bar{t} / 2$ & $\bar{P}=8 / 27\left(0.5-\frac{\bar{x}-1}{\bar{t}-2}\right)^{3}$ \\
\hline 5 & $\bar{x}_{d e}=2-\bar{t} / 2$ & $\bar{P}=8 / 27(4 / \bar{t})^{3}$ \\
\hline 6 & $\bar{x}_{c e}=\bar{t} / 2+\sqrt{\bar{t}} / 2-2$ & $\bar{P}=8 / 27\left(\frac{\bar{x}+2}{\bar{t}}-\frac{\bar{x}-1}{\bar{t}-2}\right)^{3}$ \\
\hline 7 & $\bar{x}_{d e}=0.44$ &
\end{tabular}

The parameters of the gas behind the DW front are determined after solving the gas dynamics equations (zone $1)$; in the unloading waves they are determined by a common solution (Fig.4).

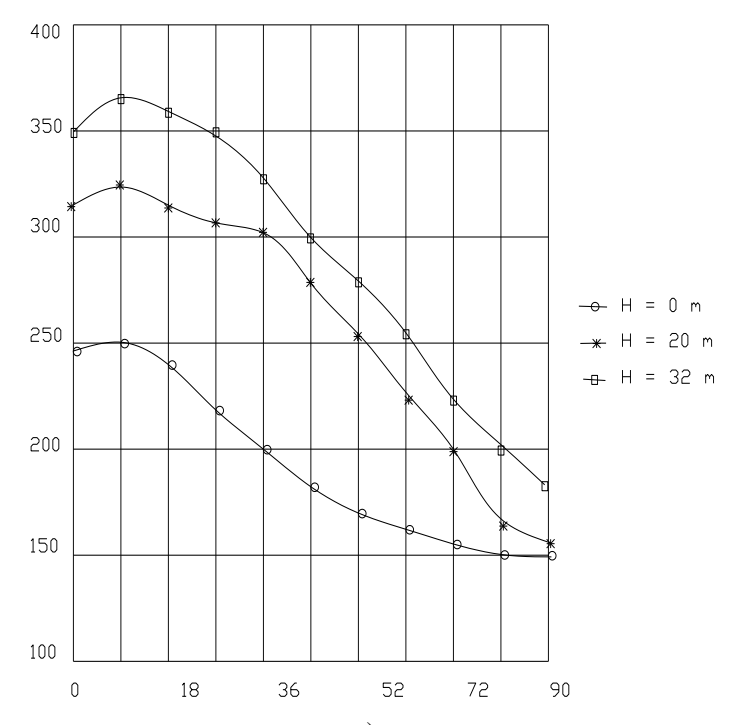

a)

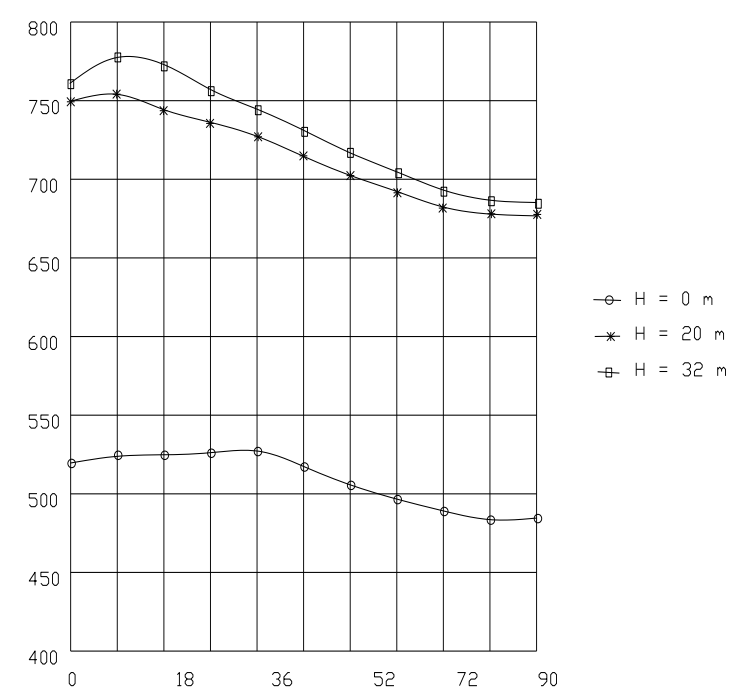

b)

Fig.4. Influence of the angle of the DW approach to the body on the relative area of target damage ( $\mathrm{a}$ - non-armored vehicles; $\mathrm{b}$ - personnel) at various heights of the warhead explosion at firing at minimum distance.

The analysis of the graphical dependences (Fig. 4) shows that the change in the angle $\psi$ within the range 90 to $9^{\circ}$ leads to an increase of the initial velocity of the fragments scattering (Table I) for the studied warhead with 26 to $35 \%$.

Additionally, the growth of the initial velocity, as shown in the graphs, facilitates the transition in charging the warheads with more powerful explosives.

The analysis of the graphical dependences shows also that with the increase of the initiating impulse velocity the angle $\psi$ is reduced, and the angle of approach of the detonation wave front to the body surface within the considered range of the initiating pulse velocities and the characteristics of the explosives contained in the warheads, represents an average $15-19^{\circ}$.

\section{CONCLUSIONS}

1. A quasi two-dimensional mathematical model is developed for describing the process of forming the initial kinematic parameters of the fragmentation field.

2. The use of system for continuous explosive initiation allows increasing the relative area of the target damage with up to $23 \%$ with varying degrees of damage.

3. The results obtained can be used in the development of ammunitions designed for countering terrorist and other threats as components of critical infrastructure protection systems.

\section{REFERENCES}

[1] K. Stoichev, A. Popov and N. Lichkov, Technologies and systems against terrorism, Bulgaria: IMSETHC-BAS, 2012.

[2] N. Georgiev, K. Stoichev, V. Pehlivanski, "Module tactical systems and means for protection developed by the IMSETHAC-BAS", Proc. Scient. Conf. of National Military University, Shumen, Bulgaria, 2014, ISSN:1314-1937.

[3] N. Georgiev, "Criteria for the quality of critical infrastructure protection systems", in Proc. Fourth National Conference with International Participation "Materials Science, Hydro- and Aerodynamics, National Security ", Bulgaria: IMSETHAC-BAS, 2014, pp 386-390.

[4] N. Georgiev, A. Kolarov, V. Pehlivanski, "One approach to selecting criteria for evaluating means of protection of objects", in Proc. International Conference on Engineering, Technologies and Systems TECHSYS 2017, Plovdiv, 2017, pp 46-52.

[5] K. Andreev and A. Belyaev, Theory of explosive substances. Moscow: Oboronprom, 1960.

[6] F. Baum, L. Orlenko, K. Stanyukovich and others. Physics of the explosion, Moscow: Nauka, 1975.

[7] V. Odintsov and T. Statsenko, "Destruction of cylinders at the wave stage", Izvestia AN SSSR, Ser. "Mechanics of a solid body", No 2, 1980 .

[8] V. Odintsov, L. Chudov and V. Selivanov, "Expansion of a thickwalled cylindrical shell under the influence of an explosive load", Izvestia AN SSSR, Ser. "Mechanics of a solid body", No 5, 1975.

[9] C. Meider, Numerical modeling of detonation, Moskow: Mir, 1985.

[10] V. Baranov, K. Velikov, I. Getsov, S. Petkov, P. Poltev and Z. Chivikov, "Wave destruction of an elasto-viscoplastic material with initial structural defects". News on the Union on the Scientists Rousse, Ser. "Technical sciences", No 3, 2001, pp. 57-62.

[11] V. Baranov, I.. Lopa, I. Getsov and S. Petkov, "Radial-wave expansion of a thick-walled homogeneous cylindrical shell", Izvestia of the Tula State University, Ser. "Problems of special mechanical engineering", Issue 4, Part 1, Russia: Tula State University, 2002, pp. 198-203.

[12] I. Getsov, S. Petkov, V. Baranov, V. Pinaev, S. Shvetsov, "Continuous initiation of a bursting charge as a way to improve the effectiveness of the warhead of burnt fire reactive projectile", Izvestia of the Tula State University, Ser. "Problems of special mechanical engineering", Issue 3, Russia: Tula State University, 2001, pp. 217223. 